\title{
Inteligencia emocional centrada en uno mismo y en el otro: Escala Rotterdam de Inteligencia Emocional (REIS)*
}

\section{Emotional intelligence centred on oneself and others: Rotterdam Scale of Emotional Intelligence (REIS)}

\author{
Pilar Teruel \\ Universidad de Zaragoza, España \\ ORCID: http://orcid.org/0000-0001-7126-524X \\ Carlos Salavera ${ }^{a}$ \\ Universidad de Zaragoza, España \\ ORCID: http://orcid.org/0000-0002-8072-2390 \\ PABLO USÁN \\ Universidad de Zaragoza, España \\ ORCID: http://orcid.org/0000-0003-3789-3781 \\ José L. Antoñanzas \\ Universidad de Zaragoza, España \\ ORCID: http://orcid.org/0000-0003-1702-3308
}

\section{a Autor de correspondencia. Correo electrónico: salavera@unizar.es}

Para citar este artículo: Teruel, P., Salavera, C., Usán, P., \& Antoñanzas, J. L. (2019). Inteligencia emocional centrada en uno mismo y en el otro: Escala Rotterdam de Inteligencia Emocional (REIS). Universitas Psychologica, 18(4), 1-12. https://doi.org/1 0.11144/Javeriana.upsy18-4.iecm

\section{RESUMEN}

El objetivo de este estudio fue la adaptación y validación al español de la Escala de Inteligencia Emocional de Rotterdam (REIS) (Pekaar, Bakker, van der Linden, \& Born, 2018) ( $N=384$, edad $=29.32$ años $)$. Se realizaron dos estudios: 1) la traducción de la escala REIS al español y se evaluó su consistencia interna, estructura factorial y validez convergente; y 2) el análisis factorial confirmatorio del cuestionario. Se obtuvieron cuatro factores: evaluación emocional centrada en sí mismo, evaluación de emociones centrada en otros, regulación de la emoción centrada en uno mismo y regulación de las emociones enfocadas en los otros. El análisis factorial exploratorio mostró claramente que los factores de la escala REIS presentan una varianza acumulada del $56.07 \%$, que mostraron ser capaces de explicar la variación única de inteligencia emocional. Por su parte, el análisis factorial confirmatorio verificó la adecuación del modelo, con una estructura sustentable compuesta por los cuatro factores identificados y 28 ítems. El REIS es un instrumento fácil de comprender y rápido de completar, por lo que se considera útil para la evaluación de la inteligencia emocional en población española.

\section{Palabras clave}

inteligencia emocional; evaluación; bienestar afectivo; adaptación; validación.

\section{ABSTRACT}

The objective of this study was to adapt and validate the Scale of Emotional Intelligence of Rotterdam (REIS) (Pekaar, Bakker, van der Linden, \& Born, 2018) ( $N=384$, age $=29.32$ years). Two studies were carried out: 1) the translation of the REIS scale into Spanish was carried out and its internal consistency, factorial structure and 
convergent validity were evaluated; and 2) the confirmatory factorial analysis of the questionnaire was carried out. Four factors were obtained: self-centered emotional evaluation, evaluation of emotions centered on others, regulation of self-centered emotion and regulation of emotions focused on others. The exploratory factorial analysis showed clearly that the factors of the REIS scale have a cumulative variance of $56.07 \%$, showing being able to explain the unique variation of emotional intelligence. For its part, the confirmatory factor analysis confirms the adequacy of the model, with a sustainable model composed of the four identified factors and 28 items. The REIS is an easy instrument to understand and quick to complete, so it is considered useful for the evaluation of emotional intelligence in the Spanish population.

Keywords

emotional intelligence; evaluation; affective well-being; adaptation; validation.

La inteligencia emocional (IE) puede definirse en términos generales como el conocimiento y/ o las competencias para abordar eficazmente las emociones, con el fin de regular las conductas sociales y emocionales (Petrides, 2012; Salovey \& Mayer, 1990; Salavera, Usán, \& Jarie, 2017; Zeidner, Roberts, \& Matthews, 2002), y supone la fusión de la razón y el corazón. A lo largo de la historia, ambos conceptos se han desarrollado de forma separada, donde el término de IE es el que ha permitido integrar estos dos aspectos de la persona: la inteligencia y la emoción. El hombre, por su naturaleza biológica, psicológica y social, es un ser emocional que tiene que aprender a educar y dirigir sus emociones para que no se conviertan en un obstáculo. La virtud, como dice Aristóteles, reside en saber en qué momento, con qué propósito, de qué modo y con quién debemos emocionarnos (FernándezBerrocal \& Ramos, 2002). Las emociones influyen en nuestros pensamientos del mismo modo que nuestros procesos cognitivos influyen en nuestros estados emocionales (Tamir \& Gutentag, 2017; Zhoc, Li, \& Webster, 2016). No se trata de dos polos opuestos, sino de aspectos complementarios (Karas \& Cieciuch, 2018; Mayer, Salovey, Caruso, \& Cherkasskiy, 2011).

En las últimas décadas, el estudio de las emociones ha cambiado, y muchos investigadores han constatado cómo el estudio y conocimiento de las emociones explica el comportamiento humano de una forma más adecuada (FernándezAbascal \& Jiménez, 2000). En la actualidad, existe un debate científico en torno a dos grandes acercamientos conceptuales en el estudio de la IE: 1) los modelos teóricos de IE como habilidad, que son aquellas aproximaciones que analizan las habilidades mentales que permiten utilizar la información que nos proporcionan las emociones para mejorar el procesamiento cognitivo; 2) los modelos mixtos o de rasgos, que incluyen dentro de la IE las habilidades mentales como rasgos estables de comportamiento y de personalidad, competencias sociales e indicadores de ajuste (Fernández-Berrocal \& Extremera, 2004; Mayer, Salovey, \& Caruso, 2000; Mayer, Salovey, \& Caruso, 2008). La posición de habilidad define IE como un conjunto de habilidades relacionadas con la emoción, similares a las capacidades cognitivas (Salovey \& Mayer, 1990; Zeidner, Roberts, \& Matthews, 2008). Por el contrario, la posición de rasgo define la IE como un conjunto de rasgos relacionados con la emoción más afín a la personalidad (Petrides, 2016). Existen, por tanto, claras divergencias, según los autores, en cuanto al concepto de inteligencia emocional.

Estas dos posturas no solo difieren en su concepción de la IE, sino que también han desarrollado instrumentos distintos (pruebas de capacidad y cuestionarios de autoinforme) para evaluar sus respectivas perspectivas de la IE. Lo que resulta evidente es que las dimensiones de la IE han recibido poca atención, la mayoría de sus instrumentos no distinguen explícitamente la IE autoevaluada de la IE centrada en los otros. Por lo tanto, sigue sin estar claro qué dimensión de la IE contribuye a qué criterio. Para solventar este problema, Pekaar, Bakker, van der Linden y Born (2018) han desarrollado una escala corta y simple para medir explícitamente la IE centrada en uno mismo y en los otros, vital para desentrañar los procesos que la subyacen. Su planteamiento reside en la idea de que la IE se capta por dos dimensiones principales: evaluación y regulación de emociones, que desempeñan un papel crucial en la forma en que las personas manejan sus emociones. En la primera parte de este proceso, 
la evaluación emocional puede llamar la atención sobre la emoción sin alterar su impacto. En la segunda parte del proceso, la emoción se regula para facilitar el estado de ánimo o la interacción social. Por lo tanto, uno podría inferir que la evaluación de la emoción funciona como una condición previa para la regulación de la emoción, sin embargo, la valoración de la emoción no siempre tiene que dar como dicha regulación. Según la capacidad, la motivación y el contexto de un individuo pueden producirse diferentes reacciones.

El objetivo del presente estudio es evaluar la posibilidad de utilización del cuestionario Rotterdam de Inteligencia Emocional REIS con población hispanohablante, como instrumento de evaluación de la inteligencia emocional. En el estudio 1 se realizó la traducción de la escala REIS al español, y se evaluó su consistencia interna, estructura factorial y validez convergente. En el estudio 2 se realizó el análisis factorial confirmatorio de la escala. En línea con sus antecedentes teóricos, la hipótesis fue que el REIS sigue una estructura de cuatro factores que consiste en la evaluación de las propias emociones, la evaluación de las emociones de los otros, la regulación de las propias emociones y la regulación de las emociones de los otros.

\section{Método}

Para la adaptación de la escala REIS al español se siguió el procedimiento clásico de traducción hacia atrás (Muñiz, Elosua, \& Hambleton, 2013), que comprende los siguientes pasos:

1. Partiendo de la versión original en inglés, se ha traducido al español por dos personas bilingües, con lengua española de origen y con experiencia en literatura científica del ámbito de la evaluación psicológica. Ambas traducciones se discutieron con el equipo investigador, hasta alcanzar un consenso y generar la primera versión de la escala en español.

2. Un psicólogo con experiencia laboral en países de habla inglesa evaluó la equivalencia conceptual, la claridad y la naturalidad de cada una de las frases y opciones de respuesta de esta primera versión. Con las modificaciones oportunas, se obtuvo una segunda versión.

3. Se realizó una consulta de esta primera versión con psicólogos que trabajaban temas de inteligencia emocional.

4. La segunda versión en español se retrotradujo de nuevo al inglés por una traductora bilingüe, con lengua inglesa de origen.

5. Se realizó un prueba piloto con 50 personas, con la finalidad de evaluar la comprensión, tiempo necesario para la cumplimentación, claridad de las preguntas y adecuación de las respuestas.

6. Con el resultado de las consultas, el equipo investigador realizó una tercera versión de la escala REIS, y se aplicó para evaluar su aplicabilidad a población española.

\section{Participantes}

La muestra de la investigación se conformó para los dos estudios con 384 personas (187 hombres y 197 mujeres), la edad media de los participantes fue de 29.329 años, con un rango entre los 18 y 44 años, y desviación estándar de 5.142. En el primer estudio participaron 208 sujetos (100 hombres y 108 mujeres) y en el segundo estudio, 176 (86 hombres y 90 mujeres). La participación de todos fue voluntaria, firmaron el consentimiento informado y se respetaron las normas éticas siguiendo la Declaración de Helsinki, para cumplir los criterios éticos de investigación con seres humanos (consentimiento informado de padres y madres, profesores y alumnos y derecho de información, protección de datos personales y garantías de confidencialidad, no discriminación, gratuidad y posibilidad de abandonar el estudio en cualquiera de sus fases). Los resultados se analizaron de manera anónima. Realizado el cálculo de la representatividad de la muestra, con un nivel de confianza del $99 \%$ y un error muestral del $5 \%$, se obtuvo que la muestra final 
encuestada resultó representativa de la provincia de Zaragoza. El estudio se diseñó como un estudio retrospectivo ex post facto (Montero \& León, 2007).

\section{Tabla 1}

Distribución de la muestra en los dos estudios ( $n=$ 384)

\begin{tabular}{lrrc}
\hline Participantes & Estudio 1 & Estudio 2 & Total \\
\hline Hombres & 100 & 86 & $186(48.44 \%)$ \\
Mujeres & 108 & 90 & $198(51.56 \%)$ \\
\hline Total & 208 & 176 & \\
\hline
\end{tabular}

\section{Instrumentos}

\section{Escala Rotterdam de Inteligencia Emocional (REIS)}

Este cuestionario, creado por Pekaar et al. (2018) evalúa la inteligencia emocional mediante una escala de Likert de 5 puntos que varía de 1 (totalmente en desacuerdo) a 5 (totalmente de acuerdo), estableciendo cuatro dimensiones de evaluación emocional:

Evaluación emocional centrada en sí mismo: la medida en que las personas perciben y comprenden sus propias emociones.

Evaluación de emociones centrada en otros: la medida en que los individuos perciben y entienden las emociones de otros individuos.

Regulación de la emoción centrada en uno mismo: la medida en que los individuos regulan sus propias emociones para alcanzar un objetivo.

Regulación de las emociones enfocadas en los otros: la medida en que los individuos regulan las emociones de los demás para alcanzar un objetivo.

Cuestionario de inteligencia emocional TEIQue -SF, Trait Emotional Intelligence Questionnaire (Petrides, 2009)

Está basado en la teoría y en el modelo de la IE rasgo, en cuyo marco, la inteligencia emocional se conceptúa como un rasgo de personalidad, localizado en los niveles inferiores de las jerarquías de la personalidad. Consta de 30 ítems, con formato Likert de 7 puntos. Comprende cuatro factores: 1) bienestar, 2) habilidades de autocontrol, 3) habilidades emocionales, y 4) habilidades sociales. Posee una fiabilidad alta $(\alpha=0.83)$. En la presente investigación, la fiabilidad de la escala fue de $\alpha=0.83$.

\section{ZKPQ-50-CC (Aluja et al., 2006)}

El cuestionario ZKPQ-50-CCes una escala que evalúa mediante autoinforme la personalidad del sujeto. Compuesto por 50 ítems, en formato dicotómico (verdadero-falso). Este instrumento evalúa las diferentes dimensiones de la personalidad, siguiendo el modelo de los Cinco Alternativos de Zuckerman, Kulman, Joireman, Teta, y Kraft (1993): neuroticismoansiedad, impulsividad-búsqueda de sensaciones, actividad, sociabilidad y agresión-hostilidad. En el presente estudio, el ZKPQ-50-CCpresentó una buena fiabilidad $(\alpha=0.78)$.

Cuestionario Panas (Watson, Clark, Eु Tellegen, 1988)

La escala de Afecto Positivo y Negativo (Panas) incluye 20 ítems, 10 de los cuales se refieren al afecto positivo (AP) y 10 al afecto negativo (AN), en dos escalas tipo Likert. Todos ellos están referidos al momento de contestar la escala, y se valoran de 0 (ausencia de la emoción) hasta 5 (presencia frecuente de la emoción). Esta escala presentó en la investigación un alfa de 0.9 para el afecto positivo, y de 0.87 para el afecto negativo.

\section{Diseño y procedimiento}

Para la selección de la muestra se solicitó la colaboración de los centros universitarios mediante llamada telefónica y, una vez confirmada la participación, se elaboró la lista de centros participantes. En el momento de la entrega de cada cuestionario, se procedió a explicar a los participantes el objetivo de la investigación, y se enfatizó en la importancia que tenía la cumplimentación de todos los ítems. 
Los participantes tenían quince minutos para completar los cuestionarios que se acaban de describir. En todos los casos se les recordaba que la información recogida era anónima y confidencial.

Para el análisis estadístico de los datos se ha empleado el programa estadístico SPSS 22.0. Comprobada la normalidad de la muestra e igualdad de varianzas, se optó por técnicas paramétricas.Se realizó un análisis descriptivo para cada una de las variables. En todos los casos se trabajó con el menor nivel de significación posible, y se han considerado significativas aquellas diferencias con un valor de $p<0.05$. Los contrastes se plantearon de forma bilateral. Para el contraste de hipótesis de dos grupos se utilizó la $t$ de Student, y para más de dos grupos la Anova unifactorial.Finalmente, con el programa estadístico AMOS v. 24, se planteó un modelo de ecuaciones estructurales que permitiese validar y cuantificar relaciones causales entre los ítems y factores de la escala REIS. Siguiendo las recomendaciones de Batista y Coenders (2000), se ha utilizado el método de estimación de máxima verosimilitud, y no el de mínimos cuadrados ponderados, dado el número de variables. Los datos recogidos en el presente estudio se obtuvieron entre los meses de octubre y noviembre de 2017.

\section{Resultados}

Los resultados se estructuraron según las dos etapas del proceso de investigación. Así, a continuación, se describen los hallazgos del estudio 1: validez de constructo, análisis de consistencia interna, validez convergente $y$ análisis factorial exploratorio (AFE); y del estudio 2, que comprende el análisis factorial confirmatorio (AFC).

\section{Estudio 1}

\section{Validez de constructo}

Elobjetivode la investigación es validar el cuestionario REIS (Pekaar et al., 2018). Tras realizar los procesos de traducción, el primer paso fue estudiar la validez de constructo.

Se empleó el método de Componentes Principales con rotación Varimax, una vez comprobada la viabilidad del análisis factorial con los siguientes criterios: la matriz de correlaciones presentó un gran número de las mismas $(87.7 \%)$, con un valor superior a 0.3 , con determinante igual a 0.002 , donde el resultado del test de esfericidad de Bartlett mostró que las variables no eran independientes (Prueba Bartlett $=4756.758, p<0.001)$. El test de Kaiser Meyer Adequacy (KMO) de adecuación de la muestra fue de 0.863, lo que indica que las correlaciones entre parejas de variables pueden ser casi explicadas por las restantes variables. Todos los valores del Measures of Sampling Adequacy (MSA) se encontraban por encima de 0.8. Estos indican que es adecuado realizar un análisis factorial de la matriz de correlaciones. Como se aprecia en la Tabla 2, se obtuvieron cuatro factores con eigenvalue mayor que 1, tomando como criterio asignar un ítem al factor en el que presentara una carga factorial mayor de 0.4, que explicó el $56.074 \%$ de la varianza total. En el factor 1, correspondiente a evaluación de las propias emociones, se encontró la mayor saturación (15.998\%), seguido del factor 2, evaluación de las emociones de los otros (14.543\%), los factores regulación de las propias emociones $(11.125 \%$ ) y regulación de las emociones de los otros (14.407\%).

Tabla 2

Porcentaje de varianza explicado por los cuatro factores de la escala REIS

\begin{tabular}{lrrr}
\hline Componentes & Autovalor & \% de la varianza & \% varianza acumulada \\
\hline Factor 1 & 4.48 & 15.998 & 15.998 \\
Factor 2 & 4.072 & 14.543 & 30.541 \\
Factor 3 & 4.034 & 14.407 & 44.948 \\
Factor 4 & 3.115 & 11.125 & 56.074 \\
\hline
\end{tabular}


La matriz de componentes extraídos mediante el análisis de componentes principales muestra los factores resultantes y los ítems del cuestionario incluidos en cada uno de ellos, así como sus saturaciones correspondientes. Se refleja la mayor saturación de cada ítem (Tabla 3). Para poder realizar comparaciones, se optó por utilizar el planteamiento que define el ajuste de un modelo a los datos como bueno, si la razón entre chi cuadrado y los grados de libertad no supera el valor de 3 (Hu \& Bentler, 1999). En la investigación, las escalas mostraron un valor menor de tres, lo que indica su buen ajuste y su validez interna.

Tabla 3

Matriz de componentes principales

\begin{tabular}{|c|c|c|c|c|}
\hline \multirow{2}{*}{ Ítems } & \multicolumn{4}{|c|}{ Factor } \\
\hline & 1 & & 3 & 4 \\
\hline \multicolumn{5}{|l|}{ Evaluación de las propias emociones } \\
\hline Siempre sé cómo me siento & 0.772 & & & \\
\hline Puedo distinguir bien mis propias emociones & 0.88 & & & \\
\hline Soy consciente de mis propias emociones & 0.853 & & & \\
\hline Entiendo por qué me siento de la manera en que me siento & 0.753 & & & \\
\hline Sé cuáles son las emociones que experimento & 0.872 & & & \\
\hline Principalmente soy capaz de explicar exactamente cómo me siento & 0.693 & & & \\
\hline $\begin{array}{l}\text { Puedo juzgar bien si los acontecimientos me tocan emocionalmente } \\
\text { Evaluación de las emociones de los otros }\end{array}$ & 0.4 & & & \\
\hline Soy consciente de las emociones de las personas que me rodean & & 0.76 & & \\
\hline Sé qué sentimientos experimentan los demás & & 0.774 & & \\
\hline Cuando miro a otras personas puedo ver cómo se sienten & & 0.636 & & \\
\hline Puedo empatizar con las personas que me rodean & & 0.658 & & \\
\hline Entiendo por qué otras personas se sienten como se sienten & & 0.745 & & \\
\hline Puedo distinguir bien entre las emociones de otras personas & & 0.803 & & \\
\hline $\begin{array}{l}\text { Puedo juzgar bien si los acontecimientos tocan a los demás } \\
\text { emocionalmente }\end{array}$ & & 0.604 & & \\
\hline \multicolumn{5}{|l|}{ Regulación de las propias emociones } \\
\hline Tengo el control de mis propias emociones & & & 0.486 & \\
\hline Puedo suprimir mis emociones fácilmente & & & 0.721 & \\
\hline No dejo que mis emociones controlen mi vida & & & 0.664 & \\
\hline Solo muestro mis emociones cuando es apropiado & & & 0.729 & \\
\hline Incluso cuando estoy enfadado/a puedo mantener la calma & & & 0.664 & \\
\hline Si quiero, pongo cara de póker & & & 0.603 & \\
\hline & & & 0.771 & \\
\hline \multicolumn{5}{|l|}{ Regulación de las emociones de los otros } \\
\hline Puedo hacer que otra persona se sienta diferente & & & & 0.769 \\
\hline Puedo alterar el estado emocional de otra persona & & & & 0.804 \\
\hline Puedo aumentar o atenuar las emociones de los demás & & & & 0.834 \\
\hline Tengo una gran influencia sobre cómo se sienten los demás & & & & 0.782 \\
\hline Sé qué hacer para mejorar el estado de ánimo de las personas & & & & 0.639 \\
\hline Sé cómo influir en las personas & & & & 0.786 \\
\hline Puedo calmar a otros & & & & 0.493 \\
\hline
\end{tabular}

\section{Validez convergente}

A continuación, se examinó la validez convergente del cuestionario. Para ello, se analizaron los diferentes componentes de los factores, con la intención de comprobar la existencia de relaciones significativas entre las dimensiones de la escala: evaluación de las propias emociones, evaluación de las emociones de los otros, regulación de las propias emociones y regulación de las emociones de los otros. Se calcularon los coeficientes de correlación de Pearson entre los factores del cuestionario REIS (Tabla 4). La validez convergente del instrumento esperaba obtener -y obtuvo- correlaciones positivas y estadísticamente significativas entre las dimensiones del instrumento, por lo que se puede afirmar que, en este caso, las medidas convergen, y se puede comprobar que los constructos que se esperaba que estuviesen relacionados, lo están.

\section{Tabla 4}

Correlaciones de Pearson entre los factores de la escala REIS

\begin{tabular}{lrrrr}
\hline \multicolumn{1}{c}{ Factor } & \multicolumn{1}{c}{1} & 2 & 3 & 4 \\
\hline Evaluación de las propias emociones & 1 & & & \\
Evaluación de las emociones de los otros & $0.314^{* *}$ & 1 & & \\
Regulación de las propias emociones & $0.21^{* *}$ & $0.071^{* *}$ & 1 & \\
Regulación de las emociones de los otros & $0.207^{* *}$ & $0.35^{* *}$ & $0.221^{* *}$ & 1 \\
\hline
\end{tabular}

La escala REIS (Pekaar et al., 2018) pretende medir explícitamente la IE centrada en uno mismo y en los otros, vital para desentrañar los procesos que la subyacen en su planteamiento, pues la evaluación y la regulación de las emociones desempeña un papel crucial en la forma en que las personas manejan sus emociones, ya que aspectos como la capacidad, la motivación y el contexto de un individuo pueden producir diferentes reacciones. Desde esta perspectiva, se analizaron las correlaciones de esta escala con el cuestionario TEIQ-SF (inteligencia emocional), dado que este plantea la IE desde una posición de rasgo, y la define como un conjunto de rasgos relacionados con la emoción más afín a la personalidad (Petrides, 2016). También se usa el cuestionario de personalidad ZKPQ-50-CC para evaluar la personalidad desde esta misma concepción al igual que la escala Panas-, dado que mide cuestiones implicadas en la evaluación y la regulación de las emociones, como son los afectos positivos y negativos (Tabla 5). Las dimensiones de la escala REIS con los otros instrumentos presentaron correlaciones estadísticamente significativas, las más fuertes ( $p$ $\geq 0,4)$ correspondieron a la evaluación de las propias emociones con los factores del TEIQ-SF. 
Tabla 5

Correlaciones con los factores del TEIQ-SF, Panas y ZKPQ-50-CC

\begin{tabular}{|c|c|c|c|c|c|}
\hline Escala & Dimensión & $\begin{array}{l}\text { Evaluación } \\
\text { propias } \\
\text { emociones }\end{array}$ & $\begin{array}{c}\text { Evaluación } \\
\text { emociones } \\
\text { de otros }\end{array}$ & $\begin{array}{l}\text { Regulación } \\
\text { propias } \\
\text { emociones }\end{array}$ & $\begin{array}{c}\text { Regulación } \\
\text { emociones } \\
\text { de otros }\end{array}$ \\
\hline \multirow{4}{*}{$\stackrel{d}{\frac{d}{4}}$} & Bienestar & $0.31^{* *}$ & 0.103 & $0.24^{* *}$ & $0.249^{* *}$ \\
\hline & Autocontrol & $0.294^{* *}$ & 0.062 & $0.496^{* *}$ & $0.146^{* *}$ \\
\hline & Emocionabilidad & $0.472^{2 *}$ & $0.478^{* *}$ & -0.082 & $0.174^{* *}$ \\
\hline & Sociabilidad & $0.278^{\circ *}$ & $0.257^{* *}$ & 0.092 & $0.496^{* *}$ \\
\hline \multirow{2}{*}{ 恚 } & Panas-P & $0.303^{\circ *}$ & $0.181^{* *}$ & $0.13^{\circ}$ & $0.243^{* *}$ \\
\hline & Panas-N & $-0.23^{\circ *}$ & -0.051 & $-0.331^{* *}$ & $-0.119^{*}$ \\
\hline \multirow{5}{*}{ 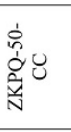 } & Ansiedad & $0.333^{\circ *}$ & -0.041 & $0.358^{* *}$ & $0.126^{*}$ \\
\hline & Impulsividad & 0.058 & -0.076 & 0.032 & $-0.163^{* *}$ \\
\hline & Actividad & $-0.19^{* *}$ & -0.103 & -0.042 & $-0.164^{* *}$ \\
\hline & Sinceridad & $-0.162^{* *}$ & $-0.163^{* *}$ & 0.028 & -0.098 \\
\hline & Agresividad & 0.089 & $0.135^{\circ}$ & $0.273^{* *}$ & -0.052 \\
\hline
\end{tabular}

\section{Fiabilidad}

Con el objetivo de comprobar la fiabilidad del instrumento, se calculó la consistencia interna de la prueba, mediante el coeficiente de Cronbach, dado que esta escala no estaba adaptada al español, y se quería analizar la adaptación que se realizó para la investigación. Este coeficiente, desde el punto de vista de la fiabilidad (Tabla 6), se puede calificar como bueno, con valores de 0.866 en el cuestionario. Los valores por encima de 0.8 se suelen considerar buenos (Cronbach, 1951), lo que indica una gran consistencia interna entre los elementos del cuestionario.

\section{Tabla 6}

Consistencia interna de los factores de la escala REIS

\begin{tabular}{|c|c|c|c|c|}
\hline Dimensiones e items & M & $D E$ & Alfa & $\begin{array}{l}\text { Alfa si se } \\
\text { elimina el } \\
\text { elemento }\end{array}$ \\
\hline Evaluación de las propias emociones & & & 0.866 & \\
\hline Siempre sé cómo me siento & 3.65 & 0.88 & & 0.86 \\
\hline Puedo distinguir bien mis propias emociones & 3.87 & 0.87 & & 0.859 \\
\hline Soy consciente de mis propias emociones & 4 & 0.83 & & 0.861 \\
\hline Entiendo por qué me siento de la manera en que me siento & 3.77 & 0.93 & & 0.859 \\
\hline Sé cuáles son las emociones que experimento & 3.92 & 0.81 & & 0.859 \\
\hline Principalmente soy capaz de explicar exactamente cómo me siento & 3.45 & 0.98 & & 0.861 \\
\hline $\begin{array}{l}\text { Puedo juzgar bien si los acontecimientos me tocan emocionalmente }\end{array}$ & 3.75 & 0.99 & & 0.864 \\
\hline Evaluación de las emociones de los otros & & & 0.857 & \\
\hline Soy consciente de las emociones de las personas que me rodean & 3.74 & 0.76 & & 0.861 \\
\hline Sé qué sentimientos experimentan los demás & 3.54 & 0.8 & & 0.861 \\
\hline Cuando miro a otras personas puedo ver cómo se sienten & 3.55 & 0.85 & & 0.86 \\
\hline Puedo empatizar con las personas que me rodean & 4.2 & 0.73 & & 0.864 \\
\hline Entiendo por qué otras personas se sienten como se sienten & 3.87 & 0.78 & & 0.863 \\
\hline Puedo distinguir bien entre las emociones de otras personas & 3.64 & 0.75 & & 0.859 \\
\hline $\begin{array}{l}\text { Puedo juzgar bien si los acontecimientos tocan a los demás } \\
\text { emocionalmente }\end{array}$ & 3.67 & 0.79 & & 0.862 \\
\hline Regulación de las propias emociones & & & 0.801 & \\
\hline Tengo el control de mis propias emociones & 3.38 & 0.94 & & 0.86 \\
\hline Puedo suprimir mis emociones fácilmente & 2.54 & 1.07 & & 0.867 \\
\hline No dejo que mis emociones controlen mi vida & 3.15 & 1 & & 0.864 \\
\hline Solo muestro mis emociones cuando es apropiado & 3.33 & 1.14 & & 0.867 \\
\hline Incluso cuando estoy enfadado/a puedo mantener la calma & 3.05 & 1.19 & & 0.865 \\
\hline Si quiero pongo cara de póker & 3.04 & 1.24 & & 0.869 \\
\hline Ajusto mis emociones cuando es necesario & 3.29 & 0.96 & & 0.86 \\
\hline Regulación de las emociones de los otros & & & 0.863 & \\
\hline Puedo hacer que otra persona se sienta diferente & 3.47 & 0.9 & & 0.86 \\
\hline Puedo alterar el estado emocional de otra persona & 3.54 & 0.86 & & 0.862 \\
\hline $\begin{array}{l}\text { Puedo aumentar o atenuar las emociones de los demás } \\
\text { Par }\end{array}$ & 3.5 & 0.8 & & 0.86 \\
\hline Tengo una gran influencia sobre cómo se sienten los demás & 3 & 0.84 & & 0.861 \\
\hline Sé qué hacer para mejorar el estado de ánimo de las personas & 3.72 & 0.74 & & 0.86 \\
\hline Sé cómo influir en las personas & 3.31 & 0.89 & & 0.858 \\
\hline Puedo calmar a otros & 3.88 & 0.76 & & 0.86 \\
\hline
\end{tabular}

\section{Estudio 2}

\section{Análisis factorial confirmatorio}

Para el estudio de la estructura interna se utilizó el análisis factorial confirmatorio, que proporciona el marco estadístico adecuado para evaluar la validez y la fiabilidad de cada ítem, en lugar de efectuar solo valoraciones globales, guiando al investigador para que optimice el proceso de construcción o adaptación del cuestionario (Batista \& Coenders, 2000).

En la Figura 1 se muestra el resultado de este análisis factorial confirmatorio (AFC) del modelo generado en el estudio exploratorio, con ecuaciones estructurales mediante el método de extracción de máxima verosimilitud, confirmando la adecuación del modelo ya que se obtuvo un modelo sustentable compuesto por los cuatro factores identificados y 28 ítems en total.

Por lo que se refiere al ajuste del modelo, los diversos índices resultaron adecuados, por lo que se puede afirmar que la estructura factorial de la escala es sustentable: $\chi^{2}(346)=621.104$; $p<0.001 ; \chi^{2} / \mathrm{gl}=1.795 ; \mathrm{CFI}=0.938 ; \mathrm{NFI}$ $=0.88 ; \mathrm{TLI}=0.928 ; \mathrm{RMSEA}=0.049, \mathrm{IC}$ $95 \%(0.043-0.055)$. La estructura factorial se analizó mediante un análisis confirmatorio en el que se planteó una estructura de cuatro factores a priori, lo que significa que los resultados del modelo son plenamente confirmatorios. Los índices mostraron un ajuste razonablemente adecuado. Los datos obtenidos en estos índices (Figura 1) permiten concluir que el modelo propuesto presenta una aproximación óptima a los datos, y puede contribuir a dar soporte a la hipótesis de la validez del cuestionario. 


\section{Figura 1}

Solución estandarizada del análisis factorial confirmatorio del cuestionario REIS para la medida de la inteligencia emocional

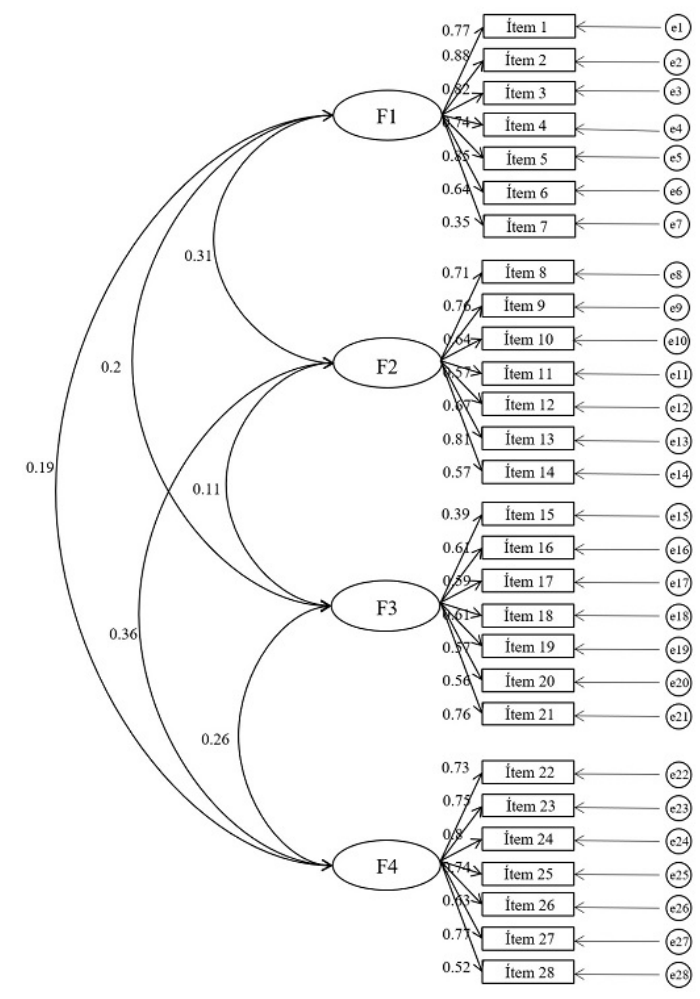

Nota. F1: Evaluación emocional centrada en sí mismo

F2: Evaluación de emociones centrada en otros F3: Regulación de la emoción centrada en uno mismo F4: Regulación de las emociones enfocadas en los otros

La Figura 1 muestra el modelo jerárquico de cuatro factores, donde los índices CFI, TLI e RFI fueron todos cercanos a 0.9, y el RMSEA fue pequeño (0.049), lo que indica que hubo un ajuste aceptable a los datos. Apoyando la segunda hipótesis (Tabla 7), el ajuste de este modelo a los datos fue significativamente mejor en comparación con un modelo jerárquico de dos factores, que representaban la evaluación y la regulación de las emociones $\left(\chi^{2}=2606.203\right.$, gl $=349, p<0.001)$ y un modelo de un factor $\left(\chi^{2}\right.$ $=10406.72, g l=350, p<0.001)$.
Tabla 7

Análisis factorial confirmatorio de la escala REIS

\begin{tabular}{lcccccc}
\hline \multicolumn{1}{c}{ Modelo } & $\chi^{2}$ & $g l$ & CFI & TLI & NFI & RMSEA \\
\hline Modelo de cuatro factores & 621.104 & 346 & 0.93 & 0.92 & 0.88 & 0.049 \\
$\begin{array}{l}\text { Modelo de dos factores } \\
\text { (evaluación y regulación) }\end{array}$ & 2606.203 & 349 & 0.69 & 0.66 & 0.68 & 0.101 \\
Modelo de un factor & 10406.72 & 350 & 0.57 & 0.55 & 0.57 & 0.122 \\
\hline
\end{tabular}

\section{Discusión}

Los objetivos del presente estudio fueron evaluar la posibilidad de utilización de la escala Rotterdam de Inteligencia Emocional (REIS) con población hispanohablante, como instrumento de evaluación de la inteligencia emocional centrada en uno mismo y en los otros, así como la realización de un análisis factorial confirmatorio de la escala.

En este estudio se ha validado la escala de inteligencia emocional (REIS), siguiendo un proceso protocolizado de traducción y adaptación, y posteriormente analizando sus propiedades psicométricas. Se presentan el procedimiento que se ha realizado para garantizar la equivalencia cultural y lingüística de la escala con su versión original, así como el análisis que apoya la consistencia interna, la validez y la estructura factorial de la misma.

Los principales resultados obtenidos tras realizar el análisis factorial del cuestionario REIS muestran una estructura formada por cuatro dimensiones, con una saturación de los ítems elevada, lo que refleja una buena consistencia interna (0.866) del cuestionario, similar a la escala original (Pekaar et al.,2018). Los cuatro factores obtenidos fueron: 1) evaluación emocional centrada en sí mismo, 2) evaluación de emociones centrada en otros, 3) regulación de la emoción centrada en uno mismo, y 4) regulación de las emociones enfocadas en los otros.

Los resultados mostraron claramente que los factores de la escala REIS presentan una varianza acumulada del $56.07 \%$. Además, se reportan evidencias a favor de la validez en relación con otras variables, ya que, por una parte, se comprobó que las puntuaciones de los factores de la escala REIS muestran una adecuada consistencia interna entre los elementos de la 
escala, lo cual, desde el punto de vista de la fiabilidad, es excelente.

Además, tras explorar la estructura factorial subyacente en el cuestionario de inteligencia emocional, se ha podido observar que en nuestro estudio se mantienen los índices de fiabilidad para cada una de las cuatro subescalas del cuestionario, ya que se obtuvieron coeficientes de fiabilidad similares a la prueba original. Se puede determinar, por consiguiente, que dicha prueba mantiene su consistencia en valoración al ser traducida al español. Se ha verificado su estructura de cuatro factores, y aportado evidencia empírica de su validez de constructo.

La escala mostró una buena validez convergente con las pruebas de inteligencia emocional, personalidad y afectos, y pretende medir los procesos que subyacen a esa IE. El estudio aportó evidencia de la relación de esta prueba con el cuestionario TEIQ-SF (inteligencia emocional), que plantea la IE desde una posición de rasgo, que indica que la escala REIS evalúa la inteligencia emocional desde una perspectiva diferente, como es la evaluación y la regulación de las emociones, pero que comparte aspectos con el TEIQ-SF, que evalúa también la inteligencia emocional, donde los factores que miden evaluación de las propias emociones y la regulación de las emociones de los otros correlacionan con todos los factores del TEIQSF, mientras que no se encontró relación con el bienestar y autocontrol en el caso de la evaluación de las emociones de otros, y con la emocionabilidad y la sociabilidad, en el caso de la regulación de las propias emociones. Esto podría indicar que los factores de evaluación de las propias emociones y la regulación de las emociones de los otros estarían más relacionados con el concepto de rasgo en la evaluación de la inteligencia emocional de la persona, que abre un nuevo campo de investigación en este sentido.

$\mathrm{Al}$ analizar las correlaciones con la prueba de personalidad, se puede concluir que el factor más relacionado con la inteligencia emocional fue la ansiedad, en concreto los factores de evaluación y regulación centrada en uno mismo, cuestión ya apuntada por los autores (Pekaar et al., 2018), y en línea con otros estudios (Nikooyeh,
Zarani, \& Fathabadi, 2017). Por tanto, se ha encontrado evidencia preliminar de las relaciones entre el escala REIS y el ZKPQ-50-CC (medida de personalidad) en lo que entendemos como un dato más a favor del escala REIS, que se revela como una herramienta corta, de fácil aplicación, fácil comprensión y adecuada para medir los constructos que se pretende. Una de las diferencias de esta escala es su soporte teórico, desde un planteamiento en el que la evaluación y la regulación de las emociones desempeña un papel crucial en la forma en la que las personas manejan sus emociones, y en el que, según la capacidad, la motivación y el contexto de un individuo, pueden producirse diferentes reacciones.

Respecto a la escala Panas, se encontró correlación de los cuatro factores del REIS con los afectos positivos, mientras que los afectos negativos correlacionaron negativamente, excepto la evaluación de las emociones de los otros con los factores del REIS, lo que indica que una mayor presencia de afectos negativos dificulta la evaluación y regulación de las emociones. Estos hallazgos dan idea de la relación entre la escala REIS, que mide cuestiones implicadas con la evaluación y la regulación de las emociones, y la escala Panas que evalúa los afectos positivos y negativos.

El modelo propuesto para la estructura factorial es sustentable, con cuatro factores identificados y 28 ítems en total, por lo que la versión traducida de la escala REIS reveló coeficientes satisfactorios con cada uno de sus cuatro factores identificados y con 28 ítems en total en su estructura factorial, por encima de los modelos planteados con dos factores (evaluación y regulación) y el modelo de un solo factor. Entendemos, por lo tanto, que estos resultados proporcionan evidencia a favor de la solidez de la estructura de esta medida y revelan que la versión española de la escala REIS consigue replicar fielmente la estructura teórica original.

En población española, la escala REIS ha revelado datos satisfactorios, ajustándose al modelo teórico subyacente y mostrando alta consistencia interna y validez. Esta escala proporciona la posibilidad de conocer no solo 
de las emociones centradas en uno mismo, sino también de las centradas en los otros, analizando la capacidad para analizar la evaluación y regulación de esas emociones. Estos aspectos se muestran claves para mantener buenas relaciones con otras personas, lo que implica, desde el planteamiento de la inteligencia emocional, el conocimiento de las competencias para abordar eficazmente las emociones a fin de regular las conductas sociales y emocionales. Por ello, la relación mostrada de las emociones con la capacidad para la comunicación efectiva —el respeto, las actitudes prosociales, la asertividad, la empatía, etc. (Batchelder, Brosnan, \& Ashwin, 2017; Bisquerra, 2009; Cook et al., 2008; Herzberg \& Brähler, 2006; Kinnaman \& Bellack, 2012; Kotsou, Leys, \& Fossion, 2018; Salavera, Usán, \& Teruel, 2019)— resultará interesante en la futura utilización del escala REIS en relación con estas variables, con estudios más avanzados, o incluso para seguir reexaminando sus propiedades.

Estos resultados deben ser interpretados teniendo en cuenta las limitaciones de este estudio donde, si bien la muestra es relevante a nivel estadístico, debería ampliarse a otros estratos de la población, donde podría darse una mayor asociación entre la inteligencia emocional y otros constructos. Serían deseables estudios longitudinales que evalúen la inteligencia emocional y permitan valorar su evolución a lo largo de un período más largo de tiempo. Además, la inteligencia emocional requiere, en muchas ocasiones, la posesión de otros tipos de habilidades, por ejemplo, cognitivas, por lo que serían interesantes estudios que evaluasen esta relación. También se podría trabajar este cuestionario con poblaciones de características especiales, como los adolescentes con ansiedad social o autismo, con aspectos diferenciales en su inteligencia emocional (Boily, Kingston, \& Montgomery, 2017; Summerfeldt, Kloosterman, Antony, \& Parker, 2006).

Como principal conclusión, la presente investigación sugiere que la inteligencia emocional en los adolescentes puede ser valorada por la escala REIS, que se ha mostrado como una medida psicométricamente eficaz, con una buena validez factorial fuerte y una adecuada consistencia interna; además de ser una herramienta breve y fácil de administrar. Aunque es necesario un mayor número de estudios e investigaciones en este sentido, los hallazgos señalan a la escala REIS como una herramienta útil para la medida de la inteligencia emocional en hispanohablantes.

\section{Referencias}

Aluja, A., Rossier, J., García L. F., Angleitner, A., Kuhlman, M., \& Zuckerman, M. (2006). A cross-cultural shortened form of the ZKPQ (ZKPQ-50-CC) adapted to English, French, German, and Spanish languages. Personality and Individual Differences, 41 (4),619-628. https://doi.org/10.1016/j.pa id.2006.03.001

Batchelder, L., Brosnan, M., \& Ashwin, C. (2017). The development and validation of the Empathy Components Questionnaire (ECQ). PLoS ONE, 12(1). https://doi.org/1 0.1371/journal.pone.0169185

Batista, J. M., \& Coenders, G. (2000). Modelos de ecuaciones estructurales. Madrid: La Muralla.

Bisquerra, R. (2009). Psicopedagogía de las emociones. Madrid: Síntesis.

Boily, R., Kingston, S. E., \& Montgomery, J. M. (2017). Trait and Ability Emotional Intelligence in Adolescents with and Without Autism Spectrum Disorder. Canadian Journal of School Psychology, 32(3-4), 282-298. https://doi.org/10.1177/0 829573517717160

Cook, C. R., Gresham, F. M., Kern, L., Barreras, R. B., Thornton, S., \& Crews, S. D. (2008). Social skills training for secondary students with emotional and/or behavioral disorders: A review and analysis of the metaanalytic literature. Journal of Emotional and Behavioral Disorders, 16(3), 131-144. https: //doi.org/10.1177/1063426608314541

Cronbach, L. J. (1951). Coefficient alpha and the internal structure of tests. Psychometrika, 16(3), 297-334 
Fernández-Abascal, E., \& Jiménez, M. P. (2000). Cien años de estudio para la emoción. Revista de Historia de la Psicología, 21(2), 707-717.

Fernández-Berrocal, P., \& Extremera, N. (2004). El papel de la Inteligencia Emocional en el alumnado: Evidencias empíricas. Revista Electrónica de Investigación Educativa, 6(2).

Fernández-Berrocal, P., \& Ramos, N. (2002). Corazones inteligentes. Barcelona: Kairos.

Herzberg, P. Y., \& Brähler, E. (2006). Assessing the Big-Five personality domains via short forms: A cautionary note and a proposal. European Journal of Psychological Assessment, 22(3),139-148. https://doi.org/10.1027/101 5-5759.22.3.139

Hu, L., \& Bentler, P. M. (1999). Cutoff criteria for fit indexes in covariance structure analysis: Conventional criteria versus new alternatives. Structural Equation Modeling: A Multidisciplinary Journal, 6(1),1-55. https ://doi.org/10.1080/10705519909540118

Karas, D., \& Cieciuch, J. (2018). The relationship between identity processes and well-being in various life domains. Personality and Individual Differences, 121, 111-119. https:/ /doi.org/10.1016/j.paid.2017.09.027

Kinnaman, J. E. S., \& Bellack, A. S. (2012). Social Skills. En W. O’Donohue \& J. E. Fisher (Eds.), Cognitive Behavior Therapy: Core principles for practice (pp. 251-272). Hoboken, NJ: John Wiley and Sons.

Kotsou, I., Leys, C., \& Fossion, P. (2018). Acceptance alone is a better predictor of psychopathology and well-being than emotional competence, emotion regulation and mindfulness. Journal of Affective Disorders, 226, 142-145. https://doi.org/10. 1016/j.jad.2017.09.047

Mayer, J. D., Salovey, P., \& Caruso, D. (2000). Models of emotional intelligence. En R. Stenberg (Ed.), Handbook of intelligence (pp. 396-420). Nueva York, NY: Cambridge University Press.

Mayer, J. D., Salovey, P., \& Caruso, D. R. (2008). Emotional intelligence: New ability or eclectic traits? American Psychologist, 63(6), 503-517.
Mayer, J. D., Salovey, P., Caruso, D., \& Cherkasskiy, L. (2011). Emotional intelligence. En J. Stenberg \& B. Kaufman (Eds.), The Cambrige Handbook of intelligence (pp. 528-549). Cambrige, MA: Cambridge University Press.

Montero, I., \& León, O. (2007). A guide for naming research studies in Psychology. International Journal of Clinical and Health Psychology, 7(3), 847-862.

Muñiz, J., Elosua, P., \& Hambleton, R. K. (2013). International Test Commission. Guidelines for test translation and adaptation ( $2^{\mathrm{a}}$ ed). Lincoln, NE: International Test Comission. https://doi.org/10.7334/psicoth $\mathrm{ema} / 2013.24$

Nikooyeh, E., Zarani, F., \& Fathabadi, J. (2017). The mediating role of social skills and sensation seeking in the relationship between trait emotional intelligence and school adjustment in adolescents. Journal of Adolescence, 59, 45-50. https://doi.org/10.1 016/j.adolescence.2017.05.012

Pekaar, K. A., Bakker, A. B., van der Linden, D., \& Born, M. P. (2018). Self- and other-focused emotional intelligence: Development and validation of the Rotterdam Emotional Intelligence Scale (REIS). Personality and Individual Differences, 120,222-233. https://doi.org/10 $.1016 /$ j.paid.2017.08.045

Petrides, K. V. (2009). Psychometric properties of the Trait Emotional Intelligence Questionnaire (TEIQue). In C. Stough, D. H. Saklofske, \& J. D. A. Parker (Eds.), The Springer series on human exceptionality. Assessing emotional intelligence: Theory, research, and applications (p. 85-101). Springer Science + Business Media. https: //doi.org/10.1007/978-0-387-88370-0_5

Petrides, K. V. (2012). Technical Manual for the Trait Emotional Intelligence Questionnaire (TEIQue). Londres: London Psychometric Laboratory.

Petrides, K. V. (2016). Four thoughts on Trait Emotional Intelligence. Emotion Review, 8(4), 345. https://doi.org/10.1177/1754073 916650504 
Salavera, C., Usán, P., \& Jarie, L. (2017). Emotional intelligence and social skills on self-efficacy in Secondary Education students. Are there gender differences? Journal of Adolescence, 60, 39-46. https://do i.org/10.1016/j.adolescence.2017.07.009

Salavera, C., Usán, P., \& Teruel, P. (2019). Contextual problems, emotional intelligence and social skills in Secondary Education students. Gender differences. Annales Medico-Psychologiques, 177(3), 223-230. https://doi.org/10.1016/j.amp.201 8.07.008

Salovey, P., \& Mayer J. D. (1990). Emotional Intelligence. Imagination, Cognition and Personality, 9, 185-211.

Summerfeldt, L. J., Kloosterman, P. H., Antony, M. M., \& Parker, J. D. A. (2006). Social anxiety, emotional intelligence, and interpersonal adjustment. Journal of Psychopathology and Behavioral Assessment, 28(1),57-68. https://doi.org/10.1007/s1086 2-006-4542-1

Tamir, M., \& Gutentag, T. (2017). Desired emotional states: their nature, causes, and implications for emotion regulation. Current Opinion in Psychology, 17, 84-88. ht tps://doi.org/10.1016/j.copsyc.2017.06.014

Watson, D., Clark, L. A. \& Tellegen, A. (1988). Development and validation of brief measures of positive and negative affect: the PANAS scales. Journal of Personality and Social Psychology, 54(6), 1063-1070.

Zeidner, M., Roberts, R. D., \& Matthews, G. (2002). Can emotional intelligence be schooled? A critical review. Educational Psychologist, 37(4), 215-231.

Zeidner, M., Roberts, R. D., \& Matthews, G. (2008). The science of emotional intelligence: Current consensus and controversies. European Psychologist, 13(1), 64-78. https://doi.org/10.1027/1016-9040. 13.1.64

Zhoc, K. C. H., LI, J. C. H., \& Webster, B. J. (2016). New reliability and validity evidence of the Emotional Intelligence Scale. Journal of Psychoeducational
Assessment, 35(6),599-614. https://doi.org/ 10.1177/0734282916653901

Zuckerman, M., Kuhlman, D. M., Joireman, J., Teta, P., \& Kraft, M. (1993). A comparison of three structural models for personality: The Big Three, the Big Five, and the Alternative Five. Journal of Personality and Social Psychology, 65(4), 757-768. https://d oi.org/10.1037/0022-3514.65.4.757

Notas

* Artículo de investigación. 